\title{
Las influencias europeas en el movimiento plástico del expresionismo-abstracto norteamericano
}

\author{
amparo Serrano de Haro Soriano *
}

La literatura crítica que gira en torno al Expresionismo-Abstracto norteamericano (que es la tendencia artística predominante en EEUU en los años 40 y 50 de nuestro siglo) tiene dos fases principales. En primer lugar una serie de libros que aparecen a partir de los años 50 y 60 de contenido laudatorio, exaltante y reafirmando lo "americano" de este arte, los títulos son por si solos extremadamente ilustrativos: Rudy Blesh Modern Art USA: Men, Rebellion, Conquest de 1956; Thomas B. Hess Abstract Painting: Background and American Phase de 1951; Irving Sandler The Triumph of American Painting: A History of Abstract Expressionism de 1970; William Seitz Abstract Expressionism Painting in America de 1955.

En segundo lugar en los años 70 y 80 una sucesión de artículos aparecidos en diversas revistas (sobre todo en Artforum) en que se cuestiona la implicación o utilización de este movimiento (que había sido siempre definido como neutral) en un sentido político, asi por ejemplo: "American Painting furing the Cold War" de Max Kozloff, de 1973; "Abstract Expressionism, weapon of the Cold War" por Eva Cockcroft de 1974; "Abstract Expressionism: the Politics of Apolitical Painting" por David y Cecile Shapiro de 1977.

\footnotetext{
- Departamento de Historia del Arte. UNED.
} 
No vamos a entrar ahora en las consideraciones y análisis que estas dos bases de literatura crítica tan compacta e interrelacionadas merecen. Pero salta a la vista que si bien la posición de la primera es defensiva frente al tema de las influencias extranjeras, y la segunda es una respuesta dirigida a ciertos aspectos de la primera, las dos soslayan el tema de las influencias. Sin embargo, es un tema que por razones obvias, está insertado en todo el desarrollo cultural norteamericano y que se encuentra aún más agudizado cuando la influencia europea empieza a ser sinónimo de cambio, de vanguardia artística, como queda patente en el famoso Armory Show.

Si bien la búsqueda de influencias toma en nuestros días un carácter totalmente distinto que en plena vigencia de los "ismos" y vanguardias en los que la originalidad que se exigía a un artista con respecto a su obra era similar a una fórmula científica o a una patente industrial; y que la evolución y los diversos factores (la conspiración de los museos, la puesta en pie de la critica formalista Greenbergiana, el despertar del mercado artístico americano) que influyeron en la aparición del movimiento Expresionista-Abstracto, exigen que se trate el tema con una cierta distancia; creemos que puede ser interesante intentar deslindar cual fue el proceso de selección, de utilización y de ocultación de esas influencias, dentro del marco artístico norteamericano.

Nos vamos a centrar esencialmente en dos puntos bien concretos: Kandinsky y los Surrealistas. Siendo las otras dos influencias importantes, Picasso y Mondrian mucho más aceptadas, la primera por ser Picasso el padre edípico de toda la pintura moderna, y Mondrian por su estrecha conexión con el grupo de los "American Abstract Artists".

La influencia de la pintura y la teoría de Kandinsky en los Expresionistas-Abstractos no se ha negado, pero sin embargo es un asunto al que no se ha dado demasiada importancia, argumentado sobre todo que los pintores norteamericanos no se sentían atraídos por la faceta espiritual y mística de los escritos de Kandinsky.

La versión más aceptada es que la influencia de Kandinsky es importante sobre todo a partir de los años 40 cuando se abre la galeria de "Non-Objective Art" (que tenía y tiene, ya que posteriormente se convirtió en el Museo Guggenheim, una importante colección de Kandiskys) y que se publica la traducción y Hilla Rebay de "Lo espiritual en el arte" en 1946. Se supone también que Hans Hofmann que en los años 1932-33 imparte clases en el "Art's Student League» de Nueva York y luego en 1934 crea su propia escuela "The Hans Hofmann School of N.Y." fue el transmisor en EEUU de las ideas Kandinskianas, como dice Stewart 
Buettner: «Hofmann (...) established himself as the foremost protagonist of German Expressionist art theory in the U.S." '. Ambos datos tienden a minimizar la posible influencia de Kandinsky al suponer que los pintores del Expresionismo-Abstracto entran en contacto con Kandinsky en una fecha tan tardía, y en la que ya se habia efectuado su maduración como artistas, como son los años 40 .

Además de que Hofmann es un artista americanizado, que tiene la curiosa caracteristica de encontrar su camino plástico por la vía abierta por los pintores Expresionistas-Abstractos, más tarde que sus propios alumnos.

Sin embargo, tenemos noticia de que anteriormente a la traducción de Hilla Rebay habian ya salido extractos de "Lo espiritual en el arte" en Camera Work, la revista de Stieglitz (aunque no son de la parte teórica de su libro sino de cuando habla de Cezanne, Matisse y Picasso) y que ya en $1914^{2}$ se había traducido "Lo espiritual en el arte", además de que en esos años se le encargó a Kandinsky un mural en Nueva York.

Es también llamativo como dos de los tratados sobre arte más conocidos de esa época, el Art Spirit de Robert Henry de 1923, y el libro de Katherine Dreier del mismo año Western Art And The New Era se encuentran referencias indirectas a Kandinsky. En el libro de Henry por via de la atención al "espíritu" creador, y a la asociación obra-vida; y el de Dreier, que tiene un cuadro de Kandinsky en la contraportada, abunda en párrafos de tipo Kandinskiano: "That is our danger today. We are once more worshipping outward forms, forgetting that it is the Spirit which we must worship and which constantly create anew, and that the only thing of real value is Spirit" ${ }^{3}$.

Pero esto son pruebas circunstanciales, lo esencial del influjo de la teoría de Kandinsky en el movimiento Expresionista-Abstracto ha sido ya puesto en evidencia por Simón Marchán en lo que es la interacción creador-obra, es decir: “el estricto paralelismo que corren la objetividad estructural de la obra (impresiones, improvisaciones, y composiciones, aquí en sentido estricto) con los procesos genéticos" ${ }^{4}$.

1 Buetrner, Stewart, American Art Theory 1945-1970. Michigan, Ann Arbor, UMI Research Press, 1981, pág. 38.

2 The Art of Spiritual Harmony. Trad. J. H. Sadler. Londres y Boston, Ed. Constable and Co. Ladt, 1914.

${ }^{3}$ Dreier, Katherine, Western Art and the New Era. An Introduction to Modern Art. New York. Brentano's Publisher 1923, pág. 12.

"Marchan, Simon, "Kandinsky y las corrientes informales", Revista de Ideas Estéticas. Madrid, núm. 104, XXVI (1968), pág. 347. 
Esto proviene de la distinción que hace Kandinsky en el epílogo de su libro De lo espiritual en el arte en que distingue entre impresiones, improvisaciones y composiciones, siendo las improvisaciones definidas como: "expresión principalmente inconsciente, generalmente súbita, de procesos de carácter interno, es decir impresión de la "naturaleza interna” 5 .

Sobre esto se apoya Marchán para decir que: "la evocación de la realidad interior o "naturaleza interna" es (...) el deseo central del artista moderno. (...) La visión interior ha encontrado en nuestros dias su radicalización" ${ }^{6}$.

Esta misma asociación entre creador, gesto-creador y obra la encontramos en Robert Henry cuando dice: "You will never draw the sense of a thing unless you aure feeling it at the time you work»?

En realidad, en nuestra opinión, la influencia de Kandinsky viene a añadirse a la corriente inglesa de fusión entre Arte y Vida que preconizan Walter Pater y Oscar Wilde.

Otra aportación en Kandinsky es la importancia que da al papel social del pintor, y su fusión entre responsabilidades políticas y artísticas. Este tema va a ser la preocupación constante del mundo artístico norteamericano de los años 30 y paralizará durante algún tiempo el avance del arte abstracto haciendo que se vuelva a lo figurativo y a temas con mensajes claros. Sin embargo, la solución a la que se acogerán los Expresionistas-Abstractos norteamericanos ya está en Kandinsky cuando dice: “La pintura es un arte, y el arte en total no es una creación inutil de objetos que se deshacen en el vacio, sino una fuerza útil que sirve al desarrollo y a la sensiblización del alma humana" ${ }^{8}$. Hacer buen arte es en sí mismo una toma de postura política y social.

Finalmente es evidente que el intento práctico-teórico de Kandinsky de convertir el arte en una ciencia objetiva dará las pautas para profundizar y valorar cada uno de los elementos plásticos que constituyen un cuadro, tanto a nivel de artistas (Ad Reinhardt, Rothko, Barnett Newman) como de críticos (Greenberg, Fried).

Es necesario también señalar como Kandinsky a través del concepto de improvisación, que basa el trabajo del artista sobre la intuición, contri-

${ }^{5}$ Kandinsky, Wasily, De to Espiritual en el Arte. Barcelona, Barral SA. Labor, 1982, pág. 121.

${ }^{6}$ Marchan, S., Opus cit., pág. 351.

' Henti, Robert, The Art Spirit. New York, Harper and Row, 1984, pág. 242.

B Kandinsky, W., Opus cit., pág. 114. 
buye a la alianza del artista con el inconsciente, lo que más tarde se desarrollará como método por excelencia de la teoria surrealista ${ }^{9}$.

Comparar los escritos de Kandinsky con los de Hofman, es un ejercicio necesario aunque obviamente son trabajos de ambiciones y calibre bien distintos. Mientras que Kandinsky ambiciona hacer una gramática del arte, una guía de la composición que posteriormente pueda ser aplicable a todas las artes, descubrir la estructura secreta del universo... y es un escrito pensado, analizado, el escrito de un verdadero teórico; Hofmann es un pintor que pone una escuela y su obra teórica Search for the Real son notas sueltas de sus clases, que daba en un inglés muy rudimentario, y que pretendian exclusivamente enseñar a pintar un cuadro.

Es evidente que las teorías de Hofman provienen de Kandinsky, inclusive su vocabulario (aunque coloquializado: tensión se convierte en "push and pull» o "tira y afloja"), y a pesar de que los conceptos sean reducidos a su significado mas pragmático, pero mantiene la noción de la obra como el resultado de la forma de sentir del pintor; sin embargo, "la necesidad interior" a la que alude Kandinsky continuamente como la clave y el principio número uno del pintor, ha desaparecido.

Han desaparecido también la tablas kandinskianas y sus diversas antinomias tanto del color como de los elementos gráficos (el punto, la línea, el plano), y aunque se alude a la gran teoría de Kandinsky de la asociación entre música y pintura, aparece sin embargo perdida en lo que es una exaltación de valores específicamente visuales y matéricamente pictóricos. Digamos que es un tratado de pintura-pintura.

Finalmente cuando Hans Hofmann menciona a la naturaleza lo hace para debatir una vez más el gastado concepto de mimesis y no para descubrir, como Kandinsky leyes comunes a la naturaleza y al arte.

Como dice Buettner: «Perhaps the most important of Hofmann's contribution to American Art in New York during the late 1930's was not his doctrinaire approach to Kandinsky's spiritual interpretation of art, but rather he is theorizing a space imbued with movements created through formal means" ${ }^{10}$.

Contrariamente al caso de Kandinsky, la influencia del surrealismo sobre el mundo cultural EEUU y en especial sobre el nacimiento del

- Puede ser también interesante ver como Marchán señala que la mayor diferencia entre Kandinsky y los pintores informalistas se basa en la visión polifocal de éstos, frente a la jerarquización del plano pictórico que aún rige en Kandisnsky.

${ }^{10}$ Buettener, Opus cit., pág. 40. 
Expresionismo-Abstracto es algo reconocido por los críticos y teóricos norteamericanos (en especial William Rubin en su libro La Escuela de Nueva York aunque todos aluden a ello) y reivindicado por muchos crítcos europeos.

No es algo sorprendente ya que aquí no se trata de especulaciones sino de hechos:

1. El éxodo de la mayoría de los artistas y teóricos surrealistas de Nueva York ( $y$ a otras zonas de América del Norte, el Caribe o América del Sur, especialmente Méjico) en la segunda Guerra Mundial.

2. La organización de revistas (V.V.V. y View en Nueva York y Dyn en Méjico) en la que se exponen y difunden sus puntos de vista.

3. El montaje de exposiciones con los principales artistas surrealistas europeos y a los que más tarde Bretón añadirán algunos surrealistas de otras nacionalidades.

Ya antes de que llegase Bretón encontramos muestras de interés por los pintores surrealistas en Nueva York, como por ejemplo la exposición de Miró ${ }^{11}$ en 1930 en la Valentine Galery, es la primera exposición de un surrealista en el continente americano. También es importante la actividad de la Galeria Julián Levy para mostrar el surrealismo, en 1932 organiza la primera exposición oficialmente surrealista: Dali, Ernst, Picasso, Man Ray, Pierre Roy, Charles Howard y Joseph Cornell. Julian Levy es también el autor del primer libro que se publicó en EEUU sobre los surrealistas ${ }^{12}$.

Aunque la exposición más importante fue la que organizó Alfred $\mathrm{H}$. Barr desde el MOMA en 1936: "Fantastic Art, Dada, Surrealism".

Podemos ver también como ya se habian empezado a hacer obras utilizando el método del automatismo, como una obra conjuntamente realizada por Baziotes, Pollock y Gerome Kamrowski en el taller de este último en el invierno de 1940, o los experimentos que se llevaban a cabo en el taller de Siqueiros.

Pero obviamente fue la llegada de Bretón la que instaló lo que podriamos llamar el reino surrealista en Nueva York. Reino que se extiende

1 El impacto de Miró es singular como prueban las numerosas exposiciones suyas que se realizan en Nueva York y las monografías que escriben sobre el Clement Greenberg ("Miró" de 1949) y James J. Sweeney que escribe el catálogo de su exposición retrospectiva de 1941 y le hace una entrevista: "Joan Miró: Comment and Interview". Partisan Review, 15, (Feb. 1948), págs. 206-212.

${ }^{12}$ LEVY, Julian, Surrealism. New York, Blak Sun, 1936. 
desde su aparición en marzo de 1941 hasta su partida en 1946 y que puede considerarse clausurada con el broche final de la "Exposition Internationale du Surrealisme" en 1947 en la Galeria Maeght. Alli están presentes las obras de todos aquellos artistas de distintas partes del mundo que Bretón consideraba surrealistas.

La llegada de Bretón a Nueva York va a suponer la reunión de los surrealistas en una serie de empresas y juegos comunes, pero también la aportación de mucho material teórico ${ }^{13}$, además organiza con Duchamp la exposición "First Papers of Surrealism" de 1942 en la Whitelaw Reid Mansion (a beneficio de los prisioneros franceses) en la que participaron Motherwell, Hare y Baziotes y sobre todo participa en la fundación de dos revistas (View 1941-47; V.V.V. junio 1942-febrero 1944) en colaboración con otros surrealistas como Duchamp, Max Ernst, Nicolas Calas, David Hare.

Todas estas actividades van a contribuir a borrar la imagen del surrealismo escandoloso que había creado Dalí cuando llegó a Nueva York por segunda vez en 1940, (su primera visita fue en 1934), donde se quedará ocho años más, aunque excluido del círculo de Bretón.

Los artistas norteamericanos comprenderán gracias a los surrealistas la importancia de crear un grupo de vanguardia con diversas actividades, y por ejemplo empezarán a crear sus propias revistas Possibilities (un solo número en 1947 coincidiendo con la desaparición de la revista View) y Tiger's Eye, también tomarán un papel más activo, como demuestra la manifestación de protesta por la política de exposiciones conservadora del "Metropolitan Museum", un cruce entre activismo político y estético tal como aparece en el Dadaismo y Surrealismo.

Los dos pilares sobre los que descansa la prueba de la influencia surrealista en EEUU son: la escritura automática y la utilización del mito.

13 Del propio Breton me parece importante señalar:

- La introducción a los catálogos: "First Papers of Surrealism" (1942) y "Art of the Century" (1942).

- "Prolégomènes à un 3eme Manifeste du Surrelaism ou non". Publicado en el primer número de la revista View en 1942.

- La conferencia pronunciada en Yale en diciembre de 1942, "Situation du Surrealism entre les deux guerres" que será publicada en V.V.V. en 1943.

- Distintos prólogos entre los cuales cabe destacar el que hace para el catálogo de Gorky en 1945 para su exposición en la Julian Levy Galery.

- La segunda edición que hace, muy ampliada, del libro Le Surrealism et la penture que finaliza con un vibrante homenaje a Gorky.

- El libro Young Cherry Trees Secure Againts Hares con dibujos de Gorky y sobre cubierta de Duchamp en 1946. 
Ahora bien la escritura automática es ante todo un concepto literario, y sólo es más tarde cuando Breton entra en contacto con la obra de Masson cuando lo desarrolla a nivel de teoría de la pintura, lo que coincide curiosamente con su estancia en EEUU. Por lo tanto la pintura automática puede ser una idea donde confluye no solo el surrealismo sino también algunos experimentos de los muralistas mejicanos (Siqueiros) y el concepto de improvisación Kandinskiana.

En cuanto al mito es sin duda una idea surrealista que jugó un papel importante, como se refleja en todas las primeras obras (fin de los años 30 y principio de los 40 ) de los artistas de la escuela de Nueva York, sin embargo, fue una temática transitoria cuya mayor importancia fue que actuó como elemento liberador hacia otros estilos.

Además los surrealistas (cuya relación con la corriente etnológica de esos años es un tema bien interesante, como queda poéticamente marcado en el hecho de que Breton llegase a Nueva York en el mismo barco que Levi-Strauss) reivindican y se interesan por primera vez por la cultura de los indios norteamericanos, lo que tendrá como resultado que los pintores norteamericanos evadirán la dependencia surrealista argumentando que es en los indios norteamericanos de donde surge su propio estilo abstracto. Este deseo de encontrar una tradición autónoma se verá legitimada por una teoría del arte propiamente norteamericana.

En definitiva, me alineo con Marcelyn Pleynet en cuanto a considerar que la influencia del arte surrealista en EEUU fue el mismo título de la que jugó el arte de Picasso o de Matisse: "No se trata de "conversión al", sino de considerar al surrealismo como un saber susceptible de dar acceso a una mayor libertad creadora (...) un arte establecido cuyos logros técnicos y estéticos hay que comprender y del que hay que sacar las mejores lecciones" "14.

Esto explicaría la falta de adhesiones totales y reales que provoca el surrealismo en los pintores norteamericanos, que habían sido aceptados dentro del grupo surrealista, tanto Motherwell («Nunca he sido un pintor surrealista porque no aceptaba el significado que daban a la imagen.» ${ }^{15}$ ) como Gorky ( EI Surrealismo es arte académico disfrazado, antiestético, sospechoso de excelencia y opuesto en gran medida al arte moderno. Sus afirmaciones de libertad son en realidad limitadoras por rígidas (...)

14 PLeynet, Marcelyn, Robert Motherwell. Paris, ed. D. Papiereski, 1989.

15 Motherwell, Robert, "The Creative Use of the Unconscious by the artist and the psychotherapist", en Annual of Psychotherapy. Volumen 5, núm. 1, 1964. 
están embriagadas de espontaneidad psiquiátrica y de sueños inexplicables") ${ }^{16}$ lo rechazan explícitamente.

Es en parte sobre ese rechazo aparente de influencias asumidas y superadas que se basa la fuerza de la «Escuela de Nueva York».

${ }^{16}$ Carta del 24 de noviembre de 1947 recogida en Mooradian, Karlen, Arshile Gorky Adoian. Chicago, Gilgamesh Press, 1978. 
